\section{The Roles and Value of}

\section{Citizen Science: Perceptions \\ of Professional Educators}

\section{Enrolled on a Postgraduate}

Course

\author{
MARIA ARISTEIDOU (1) \\ REBECCA FERGUSON (D)
}

LEIGH-ANNE PERRYMAN (D)

NATALIE TEGAMA (D)

*Author affiliations can be found in the back matter of this article
CITIZEN SCIENCE:

SPECIAL COLLECTION: CITIZEN SCIENCE IN HIGHER EDUCATION

RESEARCH PAPER

\begin{abstract}
Recent findings regarding learning in Citizen Science (CS) have led to an increase in CS project popularity in formal education classrooms. However, there has been little discussion of educators' initial views on CS. This study examined the perceptions of professional educators enrolled on a postgraduate course in relation to expertise and data quality in CS and to how CS can enhance learning. Collected data comprised comments on the FutureLearn platform made by 164 educators, over two years, after they became involved in CS activities while studying. Findings showed that, overall, educators recognise the power of CS for learning, but are sceptical about the role of experts and the quality of data gathered in this way. Further results highlighted the different motivations educators have for participating in CS (compared with other volunteers) and their need to frame learning that takes place through CS using learning theories and models. The findings of this study have considerable implications for research into designing training activities that introduce educators to CS in classroom settings and support their role as intermediaries of CS.
\end{abstract}

CORRESPONDING AUTHOR:

\section{Maria Aristeidou}

The Open University, GB

maria.aristeidou@open.ac.uk

\section{KEYWORDS:}

higher education; professional development; curriculum; student experience; formal education

\section{TO CITE THIS ARTICLE:} Aristeidou, M, Ferguson, R, Perryman, L-A and Tegama, N. 2021. The Roles and Value of Citizen Science: Perceptions of Professional Educators Enrolled on a Postgraduate Course. Citizen Science: Theory and Practice, 6(1): 24, pp. 1-14. DOI: https://doi.org/10.5334/cstp.421 


\section{INTRODUCTION}

Citizen Science (CS), which engages members of the public in the scientific process, has recently become more popular, in part because of the increasing number of easily accessible tools for accessing, sharing, and studying data (Bonney 2021). Additionally, this engagement of the public in the scientific endeavour has been recognised as a means of developing scientific knowledge and skills, and achieving behavioural changes for participants (Phillips et al. 2018; Aristeidou and Herodotou 2020; Bonney et al. 2016).

As a consequence of the reported positive impacts on learning, CS activities in formal education classrooms and spaces have increased in popularity, particularly because they can help develop a lifelong understanding of science in young people (Wyler and Haklay 2019). Current studies of CS projects in formal education report positive impacts on schoolchildren's learning, skills, and behaviour (e.g., KelemenFinan, Scheuch, and Winter 2018; (astagneyrol et al. 2020) as well as increased performance on the academic and research tasks of university students (e.g., Caruso et al. 2016; Mitchell et al. 2017). Despite the rise of CS in formal education, there has been little discussion of how educators conceive of CS, especially before they engage with it in any depth, and why they would be motivated to engage in CS projects.

Motivated by the current lack of an empirical basis for insights into educators' views on CS, we engaged professional educators (mainly educators who already teach in a classroom and have certifications) in CS activities as part of a postgraduate course in which they were enrolled, and explored their perceptions of CS. The objectives of the research presented in this paper were to examine the educators' initial conceptions of (a) whether and how CS can enhance learning and of (b) expertise and data quality in CS. This study has gone some way towards enhancing our understanding of educators' perceptions of the roles and the value of CS, knowledge that could help us design activities that attract and support educators in integrating CS in their classroom activities. The findings of this study have important implications for research into embedding CS in formal education and developing training courses for in-service and pre-service educators. This, in turn, has wider implications at a time when educators report lacking the skills and confidence to teach topics such as climate change-an area in which CS could make a significant contribution (Hazell, 2021).

\section{BACKGROUND}

This section presents areas closely aligned with CS, including learning and motivations in CS, CS in formal education, and educators as intermediaries of CS.

\section{CITIZEN SCIENCE: LEARNING AND MOTIVATIONS}

CS calls for open science communication via multiple forms of media and new forms of partnership, engaging citizens in research and opening the process of producing new knowledge. Regarding the civic value of CS, the participation of the public in the scientific endeavour empowers citizens and advances their learning and skills. Phillips et al. (2018) explored the learning objectives of more than 300 CS projects with an online presence in North America and found that $92 \%$ of those projects described at least one objective. The objectives included statements that reflect learning outcome categories concerning citizens' scientific skills (59\%) (e.g., data collection), content knowledge (28\%) (e.g., topic related learning), and the nature of science knowledge (26\%) (e.g., study design).

Systematic literature reviews of 15 empirical studies on learning outcomes in biodiversity CS projects (Peter et al. 2019) and 10 online CS projects (Aristeidou and Herodotou 2020) summarise and discuss evidence of citizen scientists' learning. The main learning outcomes reported in these reviews include citizens' changing behaviours and attitudes towards science, increased general and topic-specific science knowledge, skills acquisition, and a better understanding of the nature of science (Aristeidou and Herodotou 2020; Peter et al. 2019). More personal outcomes include ability to communicate, better language skills, increased community management ability, and greater digital literacy (Aristeidou and Herodotou 2020), as well as a sense of enjoyment and self-achievement, and an interest in nature and conservation (Peter et al. 2019), which are all important drivers for learning. These findings are, to different extents, in agreement with observations on learning objectives by Phillips et al. (2018) and demonstrate CS contributions to citizens' learning.

Learning is one of the (planned or unplanned) outcomes of participating in CS. But why do people participate in the first place? Volunteers have differentusually more than one-motivations for engaging in CS. These include personal reasons, such as interest in the topic (Aristeidou et al. 2015; Rotman et al. 2014), and altruistic factors, such as a desire to contribute to scientific research (Curtis 2015; Raddick et al. 2013). Other motivations for participation in CS include acquiring new information (Raddick et al. 2013); enjoying the research task (Aristeidou et al. 2015; Curtis 2015; Raddick et al. 2013); and sharing the same goals and values as the project (Rotman et al. 2014; Golumbic, Baram-Tsabari, and Fishbain 2020). Other important drivers include helping others and feeling part of a team (Curtis 2015; Raddick et al. 2013), and receiving recognition and feedback for contributions (Curtis 2015). 
The wealth of learning gains and incentives for participating in CS has meant that this approach has been taken up in some formal education classrooms.

\section{CITIZEN SCIENCE IN FORMAL EDUCATION}

Beyond promoting science and other forms of learning, CS has the potential to foster a lifelong interest in science in the young people who participate. Constructive exposure to science as young volunteers can positively affect them in adulthood because they develop positive attitudes and a sense of responsibility towards scientific issues (Jenkins 2011). Makuch and Aczel (2018) explain that this positive exposure can be achieved with young people's participation in exciting CS projects that develop their self-confidence and environmental agency.

Gommerman and Monroe (2017) draw attention to the ways in which engaging students in CS could also contribute to addressing inequalities in education, giving as an example the use of CS activities in the natural world as a replacement for high-cost laboratory equipment. In addition, engaging students in formal education with authentic science can also address inequalities in CS projects. The nature of mandatory curriculum-based CS projects offers the opportunity to engage participants with diverse characteristics and underserved and minority students (Bonney et al. 2016) in learning about the scientific process and the importance of scientific integrity.

Several projects involving schoolchildren in CS have already explored and reported positive impacts on their scientific knowledge and skills (e.g., Kelemen-Finan, Scheuch and Winter 2018; Castagneyrol et al. 2020), their attitudes towards science (e.g., Kelemen-Finan, Scheuch and Winter 2018; Castagneyrol et al. 2020), their ability to understand and follow a scientific protocol (e.g., Castagneyrol et al. 2020), and their motivations to take part in science (e.g., Kelemen-Finan, Scheuch, and Winter 2018). Cincera and Maskova (2011), who evaluated the implementation of CS programmes in schools and its impact on the pupils' research skills, report that results cannot be attributed solely to programme implementation, but also to teachers' level of skills and commitment.

Nevertheless, the accuracy and overall quality of data gathered by beginners and young people are often called into question (Parrish et al. 2019). To address this issue, the development of quality assessment tools alongside other new technologies for scientific collaboration-for example, the expert review (Wiggins and Crowston, 2011)-made scientific knowledge more easily accessible by scaffolding participants' contributions (even those of school students), and ensuring their quality.

\section{CONCEPTUAL FRAMEWORK: EDUCATORS AS INTERMEDIARIES OF CITIZEN SCIENCE}

Embedding CS in formal education teaching activities can benefit and help sustain the research practice because this will result in many students being introduced to and becoming accustomed to participating in CS projects (Wyler and Haklay 2019). However, opportunities to embed CS activities in formal education are limited by logistical constraints and by educators' preconceptions, motivations, and roles. Studying educators' ideas around CS can contribute to designing enhanced CS-focused professional development and training activities. Such activities, inspired by educators' views, can better support their intermediary role by emphasising the benefits of participating in CS projects with their classrooms while tackling their misconceptions and fears. Educators' increased confidence in using CS in the classroom could lead to crowds of young CS participants with a lifelong interest in scientific research.

Educators' drivers for using CS in the classroom include students' involvement in research, contribution to authentic science (Bracey 2018; Doyle et al. 2017), and contribution to their communities and the world (Bracey 2018). However, one way in which their motivations differ from those of other volunteers is the emergence of learning as a dominant motivator. Doyle, Anderson, and Boucher (2017) explain that CS can support topics that are hard to present in a classroom/lab, and can develop students' knowledge and skills with practical hands-on experience; Bracey (2018) discusses CS as a teaching tool for developing agency in students.

Educators engaging their students with CS activities could encounter challenges, such as strict curriculum objectives and timetables, and a lack of interest from students who choose not to participate (Roche et al. 2020). Research exploring the effect of different characteristics of training on 454 educators' knowledge and ability to implement CS programmes (Penuel et al. 2007) reported that offering training activities, such as discussing alignment of the programme with local standards and their own curriculum activities, led to educators feeling more prepared to support student inquiry. Other aspects that positively related to educators' preparedness were the training's focus on the programme content and the scientific process. Penuel et al. (2007) found that professional development activities were associated with increased teacher knowledge and changes to their teaching practice. Therefore, training educators as part of university-based courses could raise educators' awareness of CS at an early stage, enhance their knowledge of scientific research, and support them in their critical role as CS intermediaries.

CS as part of university-based courses is not a novel concept in science-focused fields. Studies that have 
explored CS in university classrooms report, for example, on students' positive experiences and opinions about their introduction to CS and involvement in data collection and analysis activities (e.g., Surasinghe and Courter 2012). Further, they highlight ways in which CS could enhance undergraduate education by introducing opportunities for data collection, research opportunities, and class projects (e.g., Oberhauser and LeBuhn 2012). Other studies report increased score performance and critical thinking in students within the CS experimental group, compared with those of the control lab group (e.g., Caruso et al. 2016); and increased environmental engagement and performance of students who conducted research projects on datasets contributed by citizens (e.g., Mitchell et al. 2017).

These previous studies identify educators' motivations and challenges in CS and show how CS, as part of universitybased courses, can support educators as citizen scientists. However, we know little about educators' initial perspectives on whether and how this approach can enhance learning, and their views on how participation can support research and science. These initial views on CS could inform the design of training activities for educators that would address their needs and any potential misconceptions. In this study, we explored educators' impressions of CS after engaging them in CS activities as part of a postgraduate course.

\section{METHODS COURSE DESCRIPTION}

The study was carried out with a cohort of 164 postgraduate students (110 in 2019 and 54 in 2020) from different countries. The students enrolled on a 600-hour (60 UK postgraduate credits) distance-learning course at The Open University (OU), H880 Technology-Enhanced Learning Foundations and Futures, on the FutureLearn platform. H880 was developed using the conversational learning pedagogy that underpins the design of the FutureLearn platform (Sharples and Ferguson 2019). This approach foregrounds opportunities to build shared understanding through conversation, including opportunities for asynchronous discussion alongside each activity and each piece of study material. The course description identifies the main target group of this course as (in-service) educators. Evidence from the course suggests that most of these students had little or no previous experience with CS. The eightmonth course runs annually and focuses on four types of technology-enhanced learning: CS, learning at scale, open learning, and mobile learning. Several of the activities in the course prompt students to engage in CS activities for two weeks (approximately 20 hours of full-time study per week), and share their ideas and views on the approach.

\section{Task 1: Citizen Science and learning}

The course introduces CS using a short video produced by the United States National Aeronautics and Space Administration (NASA). In this first activity, we ask students to note what they think is the most important way (if any) in which CS can enhance learning, and briefly explain why.

\section{Task 2: Expertise and data quality in Citizen Science} In Task 2 we ask students to explore two popular CS online sites: iSpot (https://www.ispotnature.org/), which is a biodiversity CS platform, and Zooniverse (https://www. zooniverse.org/), which is a platform that hosts many CS projects). This task is accompanied by an introduction to the broader field of open science. Students are asked to engage with these CS platforms by exploring the platform features and contributing to the activities. Then we ask students to discuss their ideas about expertise and data quality in CS with their fellow students on FutureLearn.

\section{DATA COLLECTION AND ANALYSIS}

In this study, we selected an exploratory sequential mixed method research design (Creswell and Plano Clark, 2018) to explore and understand educators' initial views on CS. Contributions to course discussions were first collected via the FutureLearn course log files and analysed. Then, we used the identified themes to drive the network analyses and development of graph visualisations that further explore the interconnectedness of educators' perceptions.

\section{Data collection}

Student (participant) comments in each task were retrieved from the FutureLearn platform in CSV format. The total number of comments were:

- Task 1: 206 comments (165 from 2019 and 41 from 2020) made by 113 participants and 2 tutors, and

- Task 2: 112 comments (89 from 2019 and 23 from 2020) made by 57 participants and 2 tutors.

After excluding comments by tutors and follow-up comments without content significant to this research, the final numbers were:

- Task 1: 139 comments by 107 participants, and

- Task 2: 76 comments by 57 participants.

\section{Content analysis}

We used content analysis, making valid inferences from participant comments by focusing on the meaning in context (Krippendorff 2018). Initially, Author A went through the comments ascribing descriptive codes to each student comment. The codes represented participants' 
perspectives in relation to the questions set in Task 1 and Task 2. By coding the data this way, we identified frequencies and patterns used to construct exclusive categories (themes). These themes were aligned to the main topics (discussed in the literature review), including motivation, data quality, and learning. An early inter-rater reliability agreement was calculated according to Miles, Huberman, and Saldana (2019) by dividing the number of times two coders agreed by the total number of times coding was possible; the inter-rater percentage agreement on 20 participant comments was $67 \%$ between Authors A and $B$, and $74 \%$ between Authors A and C. The three coders resolved disagreements by merging codes with similar meanings into a single theme, and creating new codes in some instances, so that the developed themes together tell a coherent story (Braun and Clarke 2006). A second interrater reliability agreement between Author $A$ and Author D on 20 participant comments (10 for each task) reached 84\% for Task 1 and 91\% for Task 2. Once we had coded all data and categorised them in themes, we calculated the frequency of each code and each theme.

\section{Data visualisation}

To explore the interconnectedness of codes and to generate conclusions on participants'/in-service educators' perceptions on (a) the learning benefits of CS and on (b) the open-access form of CS, a network analysis approach was taken. Network analysis conceptualises resources or individuals as nodes, connected by ties if a link exists between them (Kadushin 2013). In this study, the nodes represent the codes (rather than the students), and the ties represent connections between two codes. An undirected tie is present between two nodes if they are used together in one or more comments. For instance, self-efficacy, high impact, and contribution are codes assigned together to a comment (Comment A). Therefore, undirected ties connect these three codes in pairs (Figure 1). However, self-efficacy also appears a second time in Comment B, and thus its node will be bigger and connected to access, which is also used in the same comment (Table 1).

The list of codes for each participant comment was arranged to make pairs of co-used codes. Codes without a pair were presented on the graph, but without ties to other codes. Duplicates were allowed in order to produce a weighted graph showing the importance of the link between the two codes. The data were then imported into the Gephi visualisation tool in a spreadsheet, creating two undirected networks (one for each task). The network for Task 1 consists of 31 nodes (codes) and 241 ties, and the network for Task 2 consists of 25 nodes and 173 ties.

The size of the nodes corresponds to their degree of centrality (the number of their ties). The bigger the node, the more times participant comments were coded that way. The ties are also weighted (thickened) according to the number of times two codes were attached to the same comment. The modularity algorithm was used to explore whether several codes were forming clusters (and therefore were more interconnected with some codes than

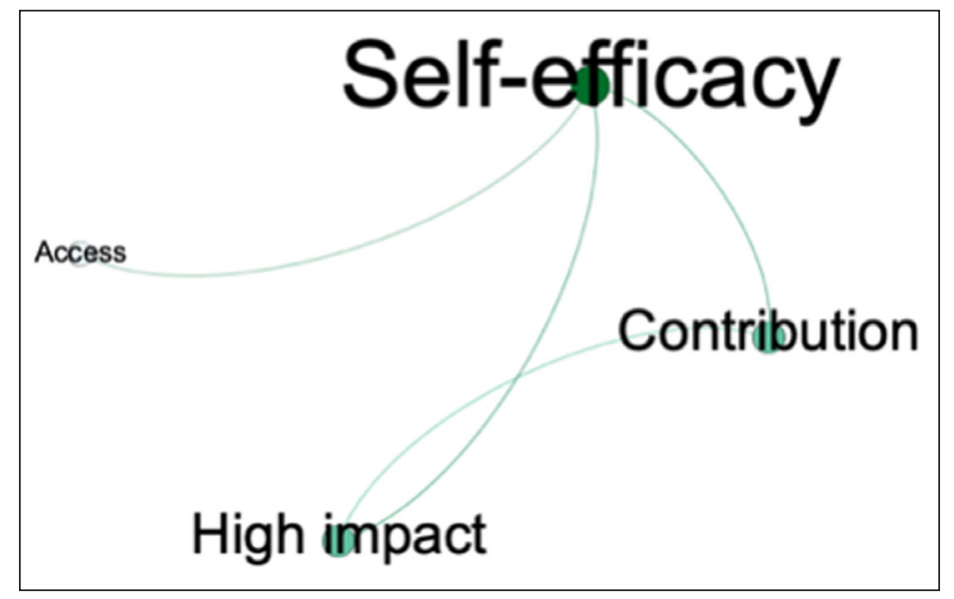

Figure 1 Example graph visualising the relationship between codes (nodes).

Comment A: "Everyone involved must have a great sense of achievement as their participation, no matter how large or small, will have contributed to significant research and findings" (Codes: self-efficacy, high impact, contribution)

Comment B: "I think the most important way in which CS enhances learning is by allowing access to new forms of learning and boosting the esteem of the participants" (Codes: self-efficacy, access)

Table 1 Example of undirected ties between two comments. 
with others) and to calculate the strength of division in clusters. The clusters are represented in different colours in the graphs.

\section{RESULTS}

\section{TASK 1: CITIZEN SCIENCE AND LEARNING}

Our coding of participants' perceptions of how CS can enhance learning is presented in Table 2. One in four comments $(25 \%)$ cites motivation as one of the most important factors that relate CS to learning. Motivation was described in terms of having an interest in the topic or being curious (personal motivation), doing real-world activities (real world), being part of something big (high impact), contributing to science and research (contribution), getting engaged in new things and directions (novelty), and gaining some reputation (qualification).

The second largest theme (groups of codes) around CS and learning was learning and agency. Twenty per cent of comments were coded this way. Participants commented on how engaging with CS could enhance understanding of the scientific process, of protocols, and of the nature of science in general (scientific research), and could help to develop scientific skills and critical thinking (skills). They also mentioned gains in supporting environmental behaviour, increasing awareness, changing attitudes towards science (behaviour and attitude), and developing feelings of research ownership and the responsibility to make changes in science and society (ownership and agency).

Reciprocity and asymmetry (R\&A) was the third theme $(16 \%)$ identified from the data. Participants in their comments discussed the design of projects and their targeted outcomes. The discussion focused on the balance between learning and science outcomes and hence the existence of some reciprocal (as opposed to asymmetrical) learning between scientists and citizens (learning R\&A). Further, participants focused on the benefits (or lack of them) that people receive from participating (benefits); the power balances between citizens and scientists in projects (power balance); expertise issues (expertise); and the participants' contributions to machine learning.

Collaboration was also widely discussed, and $14 \%$ of the comments were coded this way. Participants highlighted the significance of technology to interactions between citizens, between citizens and scientists, and with data (technology). They elaborated on how CS could develop collaboration between citizen scientists, and among scientists and science organisations (collaborations). They also explained how learning could be supported via teamwork, forum discussion, and participation in community activities (community).

\begin{tabular}{|c|c|c|}
\hline CODE & FREQUENCY & $\%$ \\
\hline Scientific research & 55 & $11 \%$ \\
\hline Learning ( $R \& A)$ & 53 & $11 \%$ \\
\hline Access & 39 & $8 \%$ \\
\hline Real world & 34 & $7 \%$ \\
\hline Personal motivation & 33 & $7 \%$ \\
\hline Technology & 31 & $6 \%$ \\
\hline Skills & 29 & $6 \%$ \\
\hline High impact & 26 & $5 \%$ \\
\hline Collaborations & 23 & $5 \%$ \\
\hline Inclusivity & 23 & $5 \%$ \\
\hline Benefits & 21 & $4 \%$ \\
\hline Contribution & 21 & $4 \%$ \\
\hline Community & 17 & $3 \%$ \\
\hline Discovery learning & 15 & $3 \%$ \\
\hline Enthusiasm & 12 & $2 \%$ \\
\hline Novelty & 8 & $2 \%$ \\
\hline Self-efficacy & 8 & $2 \%$ \\
\hline Behaviour and attitudes & 7 & $1 \%$ \\
\hline Democratising science & 7 & $1 \%$ \\
\hline Ownership and agency & 7 & $1 \%$ \\
\hline Sense of belonging & 7 & $1 \%$ \\
\hline Autonomous learning & 4 & $1 \%$ \\
\hline Communication & 4 & $1 \%$ \\
\hline Expertise & 3 & $1 \%$ \\
\hline Extracurricular & 3 & $1 \%$ \\
\hline Optional versus compulsory & 3 & $1 \%$ \\
\hline Collaborative learning & 2 & $0 \%$ \\
\hline Power balance & 2 & $0 \%$ \\
\hline Machine learning & 1 & $0 \%$ \\
\hline Qualification & 1 & $0 \%$ \\
\hline Vygotsky's ZPD & 1 & $0 \%$ \\
\hline
\end{tabular}

Table 2 Citizen science and learning: codes in a descending order of frequency.

Note: ZPD: Zone of Proximal Development.

The same percent of participant comments (14\%) focused on how CS enhances learning by providing access to science and data (access), by allowing people of all backgrounds and characteristics to take part (inclusivity), and by democratising and demystifying science and the scientific culture (democratising science). 
Wellbeing and learning models/styles were discussed in $6 \%$ of comments, a smaller amount than the previous themes. Participants explained how a sense of achievement and self-worth (selfefficacy), positive feelings such as excitement and enthusiasm (enthusiasm), communicating with others (communication), and the sense of belonging to a community (sense of belonging) could create a positive environment for learning within CS. Further, participants tried to frame the circumstances in which learning occurs within CS by discussing the mode of activities (optional versus compulsory), parental involvement in activities, and those facilitated by school clubs (extracurricular). Finally, participants attempted to characterise learning in CS by using established learning models, such as discovery learning, autonomous learning, Vygotsky's Zone of Proximal Development (ZPD), and collaborative learning.

Visualising the codes and their frequency made it easy to identify the codes used most often (degree of centrality) and where the action is. The network analysis and graph (Figure 2) revealed the codes that were more frequently applied together in participant comments and therefore highlighted the rationale behind participants' thinking about CS. Examples of codes that belong to the same group are technology and access (blue), real world and high impact (green), and communication and behaviour and attitudes (pink). Overall, the clustered groups reveal more about participants' thinking around $\mathrm{CS}$ and how it can enhance learning. Given the modularity algorithm results of 0.04 , it is noted, however, that there are no dense connections between the codes of the same cluster, and that there are more connections with codes of other clusters. Hence, we roughly described the network, but no further analysis was carried out in relation to the clusters.

From the graph, it is clear that learning (R\&A) is the code applied together with other codes the most (and therefore has the largest node size), and machine learning (bottom right) was the least co-used code. However, the most interconnected and central code (had connections with a large number of other codes) was scientific research. This had a large number of ties with learning (R\&A) (28 ties), skills (20 ties), real world (16

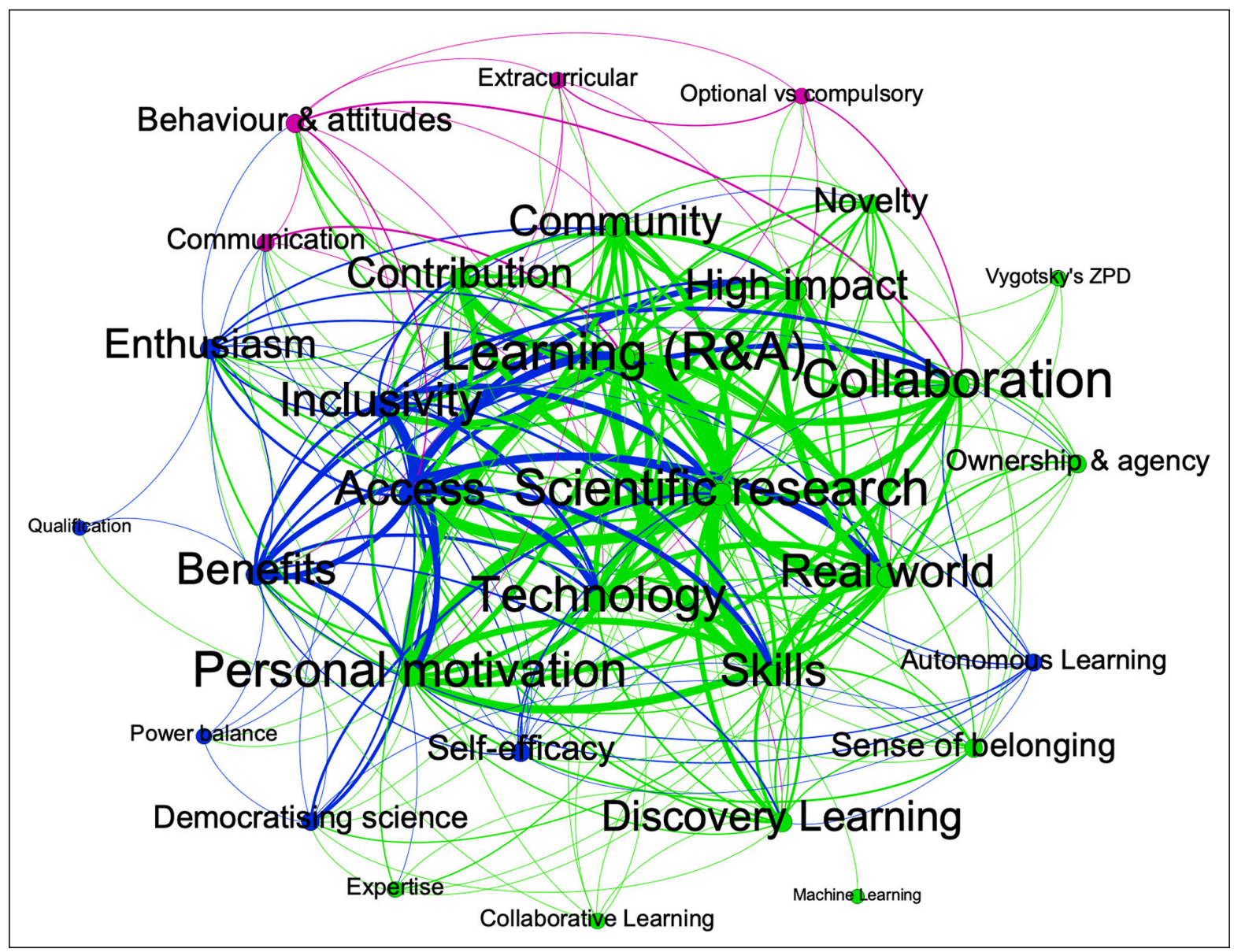

Figure 2 Citizen science and learning: clusters of codes in different colours. The size of the nodes/codes indicates how frequently a code was mentioned. 
ties), and access (16 ties). For example, in the comment below, a student explains how participation in CS can be an opportunity for people to use their knowledge in the real world:

"For those citizens who volunteer to take part in these projects, it is a good opportunity for them to put their science and maths knowledge in to practice in a real-world setting" (real world and scientific research).

Another comment focuses on how CS can promote open access by engaging people in scientific endeavours that they would not otherwise have access to:

"CS can enhance learning by getting the general public involved in scientific projects they would never normally have access to" (access and scientific research).

A large number of comments note that people's engagement with scientific research of any form can result in learning (citizens' or/and scientists'):

"The collaborative nature of many CS projects means that they can interact with the scientists who are investigating the data, while the scientists also learn from the findings of citizen scientists who help to analyse the data gathered."

Some participants, however, are more sceptical about the skills needed to take part in CS projects, even if they follow the provided scientific protocol:

"The video starts off with the premise that scientists don't have time to analyse all the data available, then quickly moves to protocols for data collection. These are two different things and require different skills. I can follow a protocol but lack the necessary skills to analyse the data, for example" (skills, scientific research).

\section{TASK 2: EXPERTISE AND DATA ACCURACY IN CITIZEN SCIENCE}

Results from coding participants' perceptions of expertise and data quality after experiencing CS activities and platforms are presented in Table 3. One in three comments (32\%) discussed expertise. Participants highlighted the importance of experts in the moderation of CS communities (expertise). They commented on the importance of verifying someone's expertise by using a reputation or ranking system (reputation) or by proving their expertise

\begin{tabular}{|c|c|c|}
\hline CODE & FREQUENCY & $\%$ \\
\hline Expertise & 48 & $17 \%$ \\
\hline Validity & 24 & $9 \%$ \\
\hline Contribution to science & 23 & $8 \%$ \\
\hline Knowledge sharing & 20 & $7 \%$ \\
\hline Credibility & 17 & $6 \%$ \\
\hline Platform & 17 & $6 \%$ \\
\hline Reputation & 16 & $6 \%$ \\
\hline Personal motivation & 15 & $5 \%$ \\
\hline Scientific research & 13 & $5 \%$ \\
\hline Database & 11 & $4 \%$ \\
\hline Verification & 11 & $4 \%$ \\
\hline Awareness & 8 & $3 \%$ \\
\hline Community & 8 & $3 \%$ \\
\hline Wisdom of crowds & 8 & $3 \%$ \\
\hline Inclusivity & 7 & $2 \%$ \\
\hline Conservation & 5 & $2 \%$ \\
\hline Data quality & 5 & $2 \%$ \\
\hline Features & 5 & $2 \%$ \\
\hline Real world & 5 & $2 \%$ \\
\hline Young people & 5 & $2 \%$ \\
\hline Reliability & 4 & $1 \%$ \\
\hline Critical mass & 3 & $1 \%$ \\
\hline Algorithm & 2 & $1 \%$ \\
\hline Interaction with scientists & 2 & $1 \%$ \\
\hline
\end{tabular}

Table 3 Expertise and data quality in citizen science: codes in a descending order of frequency.

and authority on the topic via formal qualification (credibility). They also highlighted how citizens' expertise is exploited via open science and crowdsourcing approaches in CS (wisdom of crowd).

Twenty-nine percent of participants discussed motivation in relation to the two platforms explored during the course; this was the theme discussed the second most. Participants noted, as in Task 1, that volunteers in these projects seem to have shared goals and want to contribute to science (contribution to science), have an interest in the project topic (personal motivation), want to do real-life and location-based activities (real world), want to contribute to protecting nature and the environment (conservation), and may just want to interact with scientists (interaction with scientists). 
Learning was the third-most-discussed theme (23\%), with participants expressing their ideas about how expertise can be achieved to some extent via knowledge sharing between citizens or between citizens and scientists (knowledge sharing), by getting familiar with science and scientific processes (scientific research), by developing environmental awareness (awareness), by opening access to learning via CS (inclusivity), and by engaging younger people (young people).

Data quality and its connection with the participation of non-experts in CS was coded in 13\% of participants' comments. Participants articulated their concerns with regards to the validity and accuracy of scientific data that were collected or analysed by non-scientists (validity), and the methods used in each platform for verifying the accuracy of submitted data (verification). They also discussed whether data contributed by lay people can be of good quality (data quality) and whether they can be counted on to conduct scientific work (reliability). They compared CS to Wikipedia, and mentioned the importance of reaching a large amount of data or participants for accuracy (critical mass).

Finally, participant comments focused on using technology to support expertise and data quality (12\%). Comments emphasised the significance of good user interfaces, platform structures, and moderation to scaffold lay people's contributions (platform). Further, they commented on how CS platforms can be seen as repositories of knowledge, catalogues of scientific artefacts (database), and social media platforms for knowledge exchange and for linking communities (community). Moreover, participants observed how important mapping, gamification, and quiz features are in summarising and testing newly gained citizen learning (features), and how machine learning can be used in CS platforms to support people in contributing more accurate data (algorithm).

The graph's (Figure 3) different colours (orange and purple) represent two different clusters of codes that are more interconnected than others. Examples of codes that belong to the same group are validity and expertise (orange), and interaction with scientists and features (purple). Overall, the clustered groups reveal more about participants' thinking about expertise and whether nonexperts can contribute good quality data to scientific research. As with Task 1, the modularity algorithm is 0.03 , showing no strong interconnection between the codes of the same cluster, and thus no further cluster analysis was carried out.

The graph shows that expertise and validity are the codes most frequently used with other codes (and therefore have the largest node size), and critical mass (near the bottom left) was the least co-mentioned code. Expertise was also the most interconnected and central code, and had a large number of ties with contribution



Figure 3 Expertise and data accuracy: clusters of codes in different colours. The size of the nodes/codes indicates how frequently a code was mentioned. 
to science (19 ties), validity (15 ties), credibility (13 ties), and knowledge sharing (13 ties). In the comments below, participants discuss and debate the meaning of expertise when contributing to science. For example, a participant challenged expertise on the ispot platform:

"These are genuine contributions to scientific knowledge which could come from someone with no background in scientific qualifications, therefore challenges the concept of an expert" (expertise, contribution to science).

Participants' opinions about expertise enriched the discussion. They expressed their reservations about who can be considered an expert and the validity of non-experts' data, and challenged the efficacy of such knowledgesharing spaces for scientific contribution:

"For me, I'd need to see that the contributions come from people with $\mathrm{PhDs}$ who have published articles on that topic as specialists, rather than someone's grandad taking a photo of a bug in their garden" (expertise, credibility).

"The farmer who observes nature and weather can make valid and founded predictions, as the weather expert. It is like theory and practice, they go hand in hand" (expertise, validity).

"I agree it allows for greater connectivity, sharing of enthusiasm and discussion based on common threads. It is important though to recognise the limitations regarding "expert knowledge". I would view this more as a social media platform based upon a specific interest/genre rather than an authoritative source" (expertise, knowledge sharing).

\section{DISCUSSION}

In this study, postgraduate participants, who are also professional educators, were introduced to the concept of CS and engaged in CS activities as part of a postgraduate course. Because of this, the opinions of participants in this study were influenced by their role as educators, and perhaps by their lack of previous experience with CS.

\section{CITIZEN SCIENCE AND LEARNING}

Participants' reflections on how CS can enhance learning covered many dimensions, starting with the significant role motivation plays in initiating participation. Motivators mentioned by participants corroborate previous research that cites personal motivation such as curiosity and interest in the topic (e.g., Rotman et al. 2014), altruistic factors such as contribution to science (e.g., Curtis 2015), and increased qualification and reputation (e.g., Curtis 2015). Participants also mentioned the importance of being part of something of high impact, doing real-world activities, and trying novel activities and new directions. These features are not widely mentioned in previous research. However, it is critical to emphasise that participants noted that embedding CS in classroom activities changes the voluntary nature of CS and the motivations for joining because the activities become mandatory for students. An implication of this is the need to (re)explore educators' and students' motivations for participating when designing classroom-based CS activities.

In line with previous studies that examined learning in CS (e.g., Phillips et al. 2018), participants think that participation can enhance learning by engaging people with scientific research. This may come about through following scientific protocols and through a greater understanding of scientific processes and the overall nature of science. Moreover, participants focused on how CS can develop skills (including critical thinking) and change attitudes towards science. It is important to note that agency and ownership, which are usually long-term and not very visible outcomes, were also mentioned by participants.

The most unforeseen finding, however, was the need of educators to name and frame the type of learning that takes place in CS, referring to learning theories and models. For instance, discovery learning was mentioned as reflective of the hands-on learning style encountered in CS projects. Although only a small proportion of participants mentioned learning theories and models, we consider this input significant because it has not been previously encountered in the literature. However, this outcome can be explained by the emphasis of the course on learning theories and models. This finding adds to a growing body of literature on learning in CS, which can help provide an agenda for examining evidence from a more educational perspective.

Another important participant reflection was that CS provides opportunity to participate in science regardless of background, location, age, and gender. This promotes inclusivity and could contribute to addressing inequalities in education (Gommerman and Monroe 2017). However, although there were mentions of science democratisation and the benefits (such as learning and reputation), which may be reciprocal or asymmetrical when compared with scientists, these did not correlate with long-term, more invisible outcomes, such as serving the public interest (Strasser and Haklay 2018). This apparent lack of correlation can be attributed to a possible failure of conveying such top-level outcomes in a CS project's general goal statements or to a lack of evidence demonstrating this outcome. 
Participants emphasised that an important aspect of learning via CS was the use of technology. Technology not only increases opportunities to participate in scientific activities (Wals and Peters 2017), but also allows for collaboration with others and for forming communities of interest in which people work together and learn from each other. In addition to belonging to a knowledgeexchange community, wellbeing-via the sense of belonging and communication with others-was also mentioned, highlighting benefits other than scientific and learning outcomes. Participants also stressed the role of CS in supporting people's wellbeing by developing in them a sense of enjoyment (enthusiasm) and self-achievement, a finding that is in line with previous research (Peter et al. 2019).

\section{EXPERTISE AND DATA QUALITY IN CITIZEN SCIENCE}

Reflecting on their experience with ispot and Zooniverse, participants focused on different aspects of expertise and data quality in online CS project/platforms: expertise, motivation for joining these projects/platforms, learning outcomes and processes, achieving good data quality, and the role of technology in supporting contributions and collaboration.

Participants' ideas about expertise in CS were associated mainly with the role and background of experts in the projects, and the expertise contributed by volunteers. In accordance with previous research (e.g., Lukyanenko, Parsons, and Wiersma 2016), participants expressed their concerns about expertise (and lack of it) and data accuracy in CS projects. The evidence from this study implies that participants mainly recognise expertise when this is evidenced via formal qualifications, as opposed to a reputation system. The importance of this proof of expertise is evident in the frequent mention of experts and validity together in the discussions. Although participants recognised that technology, in the form of data-validation mechanisms (algorithms), can support non-experts' contributions and allow them to take part, they doubt the accuracy and validity of these contributions.

Despite their scepticism around expertise and data quality, participants' hands-on experience with particular CS projects allowed them to recognise additional aspects in relation to motivations for participating and learning outcomes. These additions included conservation and awareness, which are related to contributing to scientific research, but are also acknowledged as participation outcomes (e.g., Peter, Diekötter, and Kremer 2019); interaction with scientists, which is not encountered in previous research; and design that allows younger people to engage.
Taken together, these results suggest that although there is an appreciation of how technology and its various features can support collaborative learning within CS, educators do not trust that these repositories of artefacts can actually promote scientific research.

\section{LIMITATIONS}

This exploratory study has examined the views professional educators have on CS after they engaged with particular activities in a postgraduate course. Although this course welcomes a diverse group of students (different locations, ages, and disciplines), the outcomes should be discussed within the context of this study and interpreted with caution. Previous knowledge or involvement in CS activities was not quantified or disclosed by the students, so their opinions cannot be attributed only to their experiences in the course. Future descriptive and causal research could better investigate the background (years of experience, discipline, etc.) of educators who engage in CS activities and relate it to their views and experiences.

\section{CONCLUSIONS}

As a result of the reported positive impacts on people's learning, CS activities in formal education classrooms and spaces have recently become more popular. Previous research, however, reports that educators face challenges, as they lack support when getting involved in CS (Roche et al. 2020). Such concerns have called into question whether CS developers need to better understand how to design classroom-friendly projects with features and tasks that attract and support educators as intermediaries of CS. This study has highlighted the views of professional educators on CS in a postgraduate course, after they were introduced to the concept and invited to get involved in certain CS activities. The contributions of this study are a further understanding of educators' conception of CS and a proposal to frame CS tasks in learning models that serve educators' needs and classroom-based activities.

Overall, the evidence from this study supports that educators perceive that CS can enhance learning. In addition to the frequently encountered learning outcomes (e.g., content knowledge, scientific skills), educators stressed the importance of less visible and long-term outcomes, such as agency, ownership, and self-achievement. The most unforeseen finding, however, was the need of educators to name and frame the type of learning that takes place in CS, referring to learning theories and models. Our results suggest several courses of action for both CS and education researchers in order to explore and categorise 
possible models of learning encountered in CS activities. These could include creating a framework that guides the categorisation of CS activities in learning models/theories and learning outcomes, signposting project activities with the framework characterisations, and developing repositories that catalogue projects based on a selected learning model or outcome.

Moreover, the findings identified several educators' motivations that are different from those of other volunteers, such as a novel way of teaching, real-world activities, interaction with scientists, and being part of something big. Research into solving this motivation mismatch and providing further evidence is already in progress, with future work focusing on examining the motivations, experiences, and challenges of educators who have already involved their classrooms in CS activities.

The findings of this study also indicate that educators who are newly introduced to CS recognise the power of CS for learning and the importance of technology to support contributions by non-experts. However, they are not convinced that data contributed to CS projects are useful or of good quality. This view may have important consequences for how and whether educators stress to younger people the importance of engaging in science. One possible way to tackle this problem will be for project owners and designers to showcase and make more visible the short-term and long-term scientific outcomes from people's contributions.

This study demonstrated educators' initial conceptions of the roles and value of CS. The findings of this study have considerable implications for research into designing training activities that introduce CS projects in classroom settings and support the role of educators as intermediaries of CS. Such activities could tackle educators' misconceptions about CS and help them address their concerns when using CS in the classroom. The training activities could involve discussions around the data quality of contributed data (validity mechanisms and outcomes from CS projects), and strategies on how it can be monitored and supported in classroom settings. Finally, the training activities could include suggestions on how to tie different CS projects and tasks to specific learning theories and models, as this may streamline the integration of CS into educators' daily lesson plans and overall curriculum aims.

\section{DATA ACCESSIBILITY STATEMENTS}

The datasets used in this study are available upon request to the corresponding author.

\section{ETHICS AND CONSENT}

The current study has been reviewed by, and received a favourable opinion from, The Open University Human Research Ethics Committee (reference number: HREC/3857/ Aristeidou)

\section{ACKNOWLEDGEMENTS}

The authors would like to acknowledge the support of National Science Foundation AISL award \#1713562 and National Science Foundation RCN-UBE award \#1919928 for covering the publication fee.

\section{COMPETING INTERESTS}

The authors have no competing interests to declare.

\section{AUTHOR CONTRIBUTIONS}

M.A.: Conceptualisation, Methodology, Formal analysis, Investigation, Original draft writing, Review, and Editing; R.F.: Conceptualisation, Review, and Editing; L.A.P: Conceptualisation, Review, and Editing; N.T.: Formal analysis, Review, and Editing.

\section{AUTHOR AFFILIATIONS}

Maria Aristeidou (D) orcid.org/0000-0001-5877-7267

The Open University, GB

Rebecca Ferguson (D) orcid.org/0000-0002-8566-8231

The Open University, GB

Leigh-Anne Perryman (D) orcid.org/0000-0002-9125-4238

The Open University, GB

Natalie Tegama (D) orcid.org/0000-0001-6515-9707

The Open University, GB

\section{REFERENCES}

Aristeidou, M and Herodotou, C. 2020. Online citizen science: a systematic review of effects on learning and scientific literacy. Citizen Science: Theory and Practice, 5: 1-12. DOI: https://doi. org/10.5334/cstp. 224

Aristeidou, M, Scanlon, E and Sharples, M. 2015. Weatherit: evolution of an online community for citizen inquiry. In: Proceedings of the 15th International Conference on Knowledge Technologies and Data-Driven Business - i-KNOW '15. ACM Press, Graz, Austria. 1-8. DOI: https://doi.org/10.1145/2809563.2809567 
Bonney, R. 2021. Expanding the impact of citizen science. Bioscience, 71: 448-451. DOI: https://doi.org/10.1093/biosci/biab041

Bonney, R, Phillips, TB, Ballard, HL and Enck, JW. 2016. Can citizen science enhance public understanding of science? Public Understing of Science, 25: 2-16. DOI: https://doi. org/10.1177/0963662515607406

Bracey, G. 2018. Teaching with citizen science: an exploratory study of teachers' motivations \& perceptions. PhD thesis. University of Missouri, St. Louis.

Braun, $\mathbf{V}$ and Clarke, V. 2006. Using thematic analysis in psychology. Qualitative Research in Psychology, 3: 77-101. DOI: https://doi.org/10.1191/1478088706qp063oa

Caruso, JP, Israel, N, Rowland, K, Lovelace, MJ and Saunders, MJ. 2016. citizen science: the small world initiative improved lecture grades and california critical thinking skills test scores of nonscience major students at florida atlantic university. Journal of Microbiology \& Bioogy Education, 17: 156-162. DOI: https://doi.org/10.1128/jmbe.v17i1.1011

Castagneyrol, B, Valdés-Correcher, E, Bourdin, A, Barbaro, L, Bouriaud, O, Branco, M, Centenaro, G, Csóka, G, Duduman, $M L$, Dulaurent, $A M$, Eötvös, CB, Faticov, M, Ferrante, M, Fürjes-Mikó, Á, Galmán, A, Gossner, MM, Harvey, D, Howe, AG, Kaennel-Dobbertin, M, Koricheva, J, Löveï, GL, Lupaştean, D, Milanovic, S, Mrazova, A, Opgennoorth, L, Pitkänen, JM, Popovic, M, Roslin, TV, Scherer-Lorenzen, M, Sam, K, Tahadlová, M, Thomas, $R$ and Tack, AJM. 2020. Can school children support ecological research? lessons from the 'oak bodyguard' citizen science project. Citizen Science: Theory and Practice, 5: 10. DOI: https://doi.org/10.5334/cstp.267

Cincera, J and Maskova, V. 2011. GLOBE in the Czech Republic: A program evaluation. Environmental Education Research, 17: 499-517. DOI: https://doi.org/10.1080/13504622.2011.557497

Creswell, J and Plano Clark, VL. 2018. Designing and Conducting Mixed Methods Research. 3rd ed. SAGE Publications Inc.

Curtis, V. 2015. Motivation to participate in an online citizen science game. Science Communication, 37: 723-746. DOI: https://doi.org/10.1177/1075547015609322

Doyle, C, Anderson, D and Boucher, M. 2017. What is online citizen science anyway? An educational perspective. Available at https://arxiv.org/abs/1805.00441 [Last accessed 28 June 2021].

Golumbic, Y, Baram-Tsabari, A and Fishbain, B. 2020. Engagement styles in an environmental Citizen Science project. Journal of Science Communication, 19: A03. DOI: https://doi.org/10.22323/2.19060203

Gommerman, L and Monroe, MC. 2017. Lessons Learned from Evaluations of Citizen Science Programs. EDIS. University of Florida.

Hazell, W. 2021. Climate change: $70 \%$ of UK teachers say they have not been properly trained to teach about crisis. iNews Education. Available at https://inews.co.uk/news/education/ climate-change-uk-teachers-training-teach-global-warmingschools-914301 [Last accessed 30 June 2021].
Jenkins, LL. 2011. Using Citizen Science beyond teaching science content: A strategy for making science relevant to students' lives. Cultural Studies of Science Education, 6: 501-508. DOI: https://doi.org/10.1007/s11422-010-9304-4

Kadushin, C. 2013. Understanding Social Networks: Theories, Concepts, and Findings. Oxford University Press.

Kelemen-Finan, J, Scheuch, M and Winter, S. 2018. Contributions from citizen science to science education: an examination of a biodiversity citizen science project with schools in Central Europe. International Journal of Science Education, 40: 2078-2098. DOI: https://doi.org/10.1080/09500 693.2018.1520405

Krippendorff, K. 2018. Content Analysis: An Introduction to Its Methodology. 4th ed. Thousand Oaks, CA: SAGE Publications Inc.

Makuch, KE and Aczel, MR. 2018. Children and Citizen Science. In: Hecker, S, Haklay, M, Bowser, A, Makuch, Z, Vogel, J and Bonn, A (eds.), Citize Science: Innovation in Open Science, Society and Policy, 391-409. London: UCL Press. DOI: https://doi. org/10.2307/j.ctv550cf2.34

Miles, MB, Huberman, MA and Saldana, J. 2019. Qualitative Data Analysis: A Methods Sourcebook. 4th ed. Beverly Hills, CA: Sage.

Mitchell, N, Triska, M, Liberatore, A, Ashcroft, L, Weatherill, R and Longnecker, N. 2017. Benefits and challenges of incorporating Citizen Science into university education. PLoS One, 12: e0186285. DOI: https://doi.org/10.1371/journal.pone.0186285

Oberhauser, $\mathbf{K}$ and LeBuhn, G. 2012. Insects and plants: engaging undergraduates in authentic research through Citizen Science. Frontiers in Ecology and the Environment, 10(6): 318-320. DOI: https://doi.org/10.1890/110274

Parrish, JK, Jones, T, Burgess, HK, He, Y, Fortson, L and Cavalier, D. 2019. Hoping for optimality or designing for inclusion: Persistence, learning, and the social network of Citizen Science. In: Proceedings of the National Academy of Sciences U. S. A., 116: 1894-1901. DOI: https://doi.org/10.1073/pnas.1807186115

Penuel, WR, Fishman, BJ, Yamaguchi, R, Associates, A and Gallagher, LP. 2007. What makes professional development effective? Strategies that foster curriculum implementation. American Educational Research Journal, 44: 921-958. DOI: https://doi.org/10.3102/0002831207308221

Peter, M, Diekötter, T and Kremer, K. 2019. Participant outcomes of biodiversity citizen science projects: a systematic literature review. Sustainability, 11: 2780. DOI: https://doi.org/10.3390/ su11102780

Phillips, T, Porticella, N, Constas, M and Bonney, R. 2018. A Framework for Articulating and Measuring Individual Learning Outcomes from Participation in Citizen Science. Citizen Science: Theory and Practice, 3: 3. DOI: https://doi.org/10.5334/cstp.126

Raddick, JM, Bracey, G, Gay, PL, Lintott, CJ, Cardamone, C, Murray, P, Schawinski, K, Szalay, AS and Vandenberg, J. 2013. Galaxy zoo: Motivations of citizen scientists. Astronomy Education Review, 12: 1-41. DOI: https://doi.org/10.3847/AER2011021 
Roche, J, Bell, L, Galvão, C, Golumbic, YN, Kloetzer, L, Knoben, N, Laakso, M, Lorke, J, Mannion, G, Massetti, L, Mauchline, A, Pata, K, Ruck, A, Taraba, P and Winter, S. 2020. Citizen science, education, and learning: challenges and opportunities. Frontiers in Sociology, 5: 14. DOI: https://doi.org/10.3389/fsoc.2020.613814

Rotman, D, Hammock, J, Preece, J, Hansen, D, Boston, C, Bowser, $\mathbf{A}$ and $\mathbf{H e}$, Y. 2014. Motivations affecting initial and long-term participation in citizen science projects in three countries. In: IConference 2014 Proceedings . iSchools. 110-124. DOI: https://doi.org/10.9776/14054

Sharples, M and Ferguson, R. 2019. Pedagogy-informed design of conversational learning at scale. In: CEUR Workshop Proceedings, 2437: Article No. 2. DOI: https://doi. org/10.1145/3330430.3333642

Strasser, B and Haklay, M. 2018. Citizen Science: Expertise, Democracy, and Public Participation. UCL Press, London. DOI: https://doi.org/10.23987/sts.60425
Surasinghe, T and Courter, J. 2012. Using eBird to integrate citizen science into an undergraduate ecology field laboratory. Bioscene: Journal of College Biology Teaching, 38(2): 16-20.

Wals, AEJ and Peters, MA. 2017. Flowers of resistance: Citizen Science, ecological democracy and the transgressive education paradigm. In: König, A and Ravetz, J (eds.), Sustainability Science: Key Issues. Routledge. DOI: https://doi. org/10.9774/gleaf.9781315620329_3

Wiggins, A and Crowston, K. 2011. From conservation to crowdsourcing: a typology of citizen science. In: 2011 44th Hawaii International Conference on System Sciences, 1-10. DOI: https://doi.org/10.1109/ HICSS.2011.207

Wyler, D and Haklay, M. 2019. Integrating Citizen Science into university. In: Citizen Science. UCL Press, London. 168-182. DOI: https://doi.org/10.2307/j.ctv550cf2.18

\section{TO CITE THIS ARTICLE:}

Aristeidou, M, Ferguson, R, Perryman, L-A and Tegama, N. 2021. The Roles and Value of Citizen Science: Perceptions of Professional Educators Enrolled on a Postgraduate Course. Citizen Science: Theory and Practice, 6(1): 24, pp. 1-14. DOI: https://doi.org/10.5334/cstp.421

Submitted: 31 March 2021 Accepted: 20 August 2021 Published: 01 December 2021

\section{COPYRIGHT:}

(C) 2021 The Author(s). This is an open-access article distributed under the terms of the Creative Commons Attribution 4.0 International License (CC-BY 4.0), which permits unrestricted use, distribution, and reproduction in any medium, provided the original author and source are credited. See http://creativecommons.org/licenses/by/4.0/.

Citizen Science: Theory and Practice is a peer-reviewed open access journal published by Ubiquity Press.

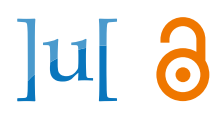

